\title{
Segmentation of overlapping/aggregating nuclei cells in biological images
}

\author{
F. Cloppet and A. Boucher \\ Crip5-Sip, Université Paris Descartes \\ 45, rue des Saints Pères 75006 Paris \\ cloppet@math-info.univ-paris5.fr
}

\begin{abstract}
This paper presents a method of overlapping/aggregating nuclei cells segmentation. This method is based on the watershed segmentation algorithm, but the specificity of this work is to introduce some prior information about the usual shape of normal/pathological nuclei cells. Such prior information will help to optimize the right set of markers, from which the flooding will be done. This approach has been implemented and tested, and the results evaluated by cell biology experts show the efficiency of the proposed approach.
\end{abstract}

\section{Introduction}

Over the past fifteen years, the field of optical imaging has been developing rapidly with the advent of a wide range of new probes (especially fluorescent ones), with the advances in microscopy (immunofluorescence light microscope, confocal microscope, video microscopy...) and image analysis. All these advances have revolutionized our knowledge and understanding of the dynamic structures and events related to living cells.

The biological background of this work is based on alterations in nuclear morphology of fibroblasts from Hutchinson-Gilford Progeria Syndrome (premature aging disease of children). The knowledge of the cellular structure that underlies nuclear morphometric changes with respect to a non pathological shape is still limited. But such changes are frequently observed in various nuclei, including several human diseases like cancer.

Cell nuclear morphology is a hot topic research in biology. Several biological functions seem to be related to major changes in the geometry of the nucleus. Nuclear size and shape are good visual descriptors of abnormal nuclear behavior, and could lead to a quick and efficient diagnosis of nucleus normality.
Highly reliable shape characterization and description[1] involves computer vision problems for which efficient solutions have not yet been found, such as extracting in a high precise way the contour of each nucleus.

Section 2 gives some details about the typical problems encountered in the images that have to be processed and deals with the problem of segmentation, while Section 3 describes our contribution to segment aggregating/overlapping nuclei. The results are presented and discussed in section 4 , and section 5 concludes the paper.

\section{Segmentation}

In this study, the images are taken with camera fixed on an immunofluorescence light microscope. In fact, the major problem with such images is that they are disturbed by out-of-focus light mostly due to the thickness of the specimen. This can lead to some overlapping areas between several nuclei since they are not on the same focal plane (see Figure 1).

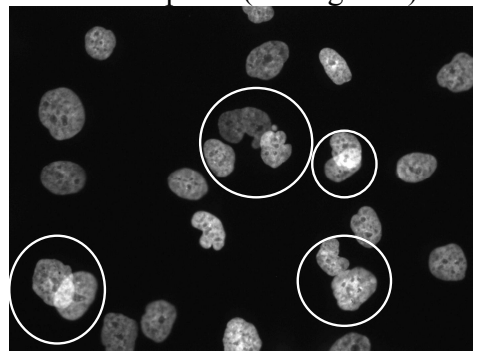

Figure 1. Nuclei of cells in an immunofluorescence image

This problem of overlapping areas could be largely solved by using confocal microscope, but the acquisition of images is much slower. Indeed, at the moment, this is hardly compatible with large screening tests of drugs. Furthermore, both sources of images (confocal or light microscope) present similar disturbances due to the auto-fluorescence of the 
medium, the acquisition noise induced by the camera detector, and various imperfections of the optical system. Therefore, the segmentation step developed to extract each nucleus has to be done with high precision.

Segmentation remains one of the most challenging process in image analysis. It refers to the task of detecting boundaries of objects of interest in an image. Several approaches have been proposed to deal with objects in contact, and they are mainly divided into two categories: energy-driven segmentation [2-3] and watershed-based segmentation [4].

Recent methods, among the energy-driven segmentation methods, based on deformable contours [5] or level sets [6], prevent the contours of the nuclei that are in contact from merging. They introduce in the energy functional an overlap penalty between each pair of contours. The multiphase level set method [7] can extract regions in contact, and overlapping regions. But all these methods are time consuming, with a lot of parameters to be tuned.

The watershed transform [4] has proven to be a powerful and fast technique for both contour detection and region-based segmentation. Some interesting properties make it useful for many different image segmentation applications: it is simple and intuitive, can be parallelized, and always produces an entire partition of the image.

It has been applied to biological images [8,9] and has given good results, especially on blurred grey level images. However, it has some drawbacks (oversegmentation, sub-segmentation, and sensitivity to noise...). This transform can be improved, in particular by the introduction of prior information [10].

The initialization step is probably the most critical step of this kind of transform. A good set of markers will avoid severe over-segmentation. This paper will focus on this problem.

\section{Segmenting aggregating/overlapping nuclei}

The proposed system is illustrated on Figure 2. The main difficulty is to first detect whether a connected component extracted on the image corresponds to a single nucleus or to a group of several nuclei. This information will be helpful for selecting the right markers in order to avoid over or sub-segmentation given by the watershed transform.

\subsection{Extraction of the right set of markers}

Overlapping/aggregating nuclei have intersecting contours in points of high concavity. To select those involved in the segmentation process among the all set of concavity points, prior information about normal or abnormal nuclei has to be used.

For example, normal nuclei have a slightly elongated but regular shape with a quite small area, while abnormal nuclei might be much bigger and/or lobulated. In fact, points of significant concavity that are extracted can correspond to aggregating or overlapping nuclei areas, but also to lobulated areas of abnormal nuclei.

In order to identify these three types of areas, segments linking significant concavity points (see Figure 3a) are explored for selecting the one(s) that correspond(s) to true separation segment(s) (see Figure 3b).

For this purpose, two templates of high biological plausibility (based on visual inspection of about 300 nuclei) have been established:

- overlapping template, - aggregating template.

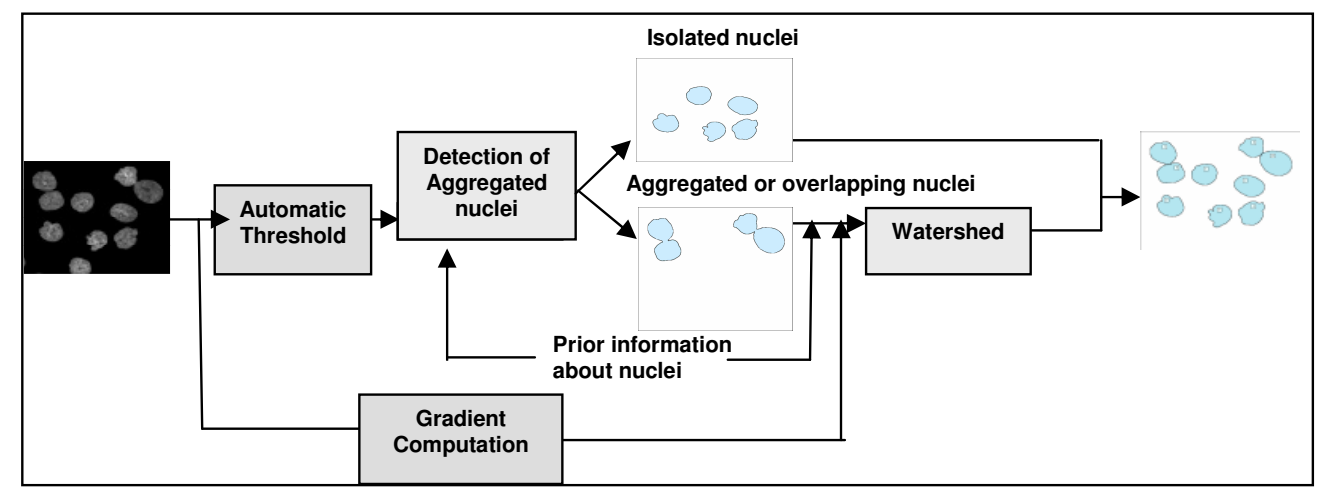

Figure 2. Illustration of the segmentation step 


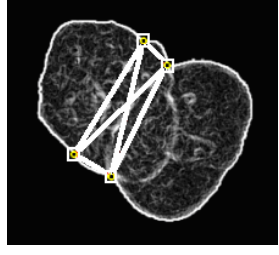

a

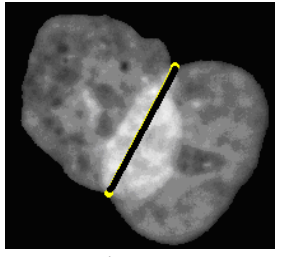

b
Figure 3. a.Segments linking significant concavity points b.Selected separation segment

The overlapping template (see. Figure 4), is based on the hypothesis that if two nuclei overlap, the connected component has to be enough elongated to contain two nuclei. $[\mathrm{AB}]$ is the potential segment of separation that connects two significant concavity points. All the points $\mathrm{C}, \mathrm{D}, \mathrm{E}, \mathrm{F}, \mathrm{G}, \mathrm{H}$ of the template have to be inside the connected component. At the moment, the distances $\mathrm{CD}, \mathrm{EF}=\mathrm{GH}$ are evaluated by observing a high number of nuclei.

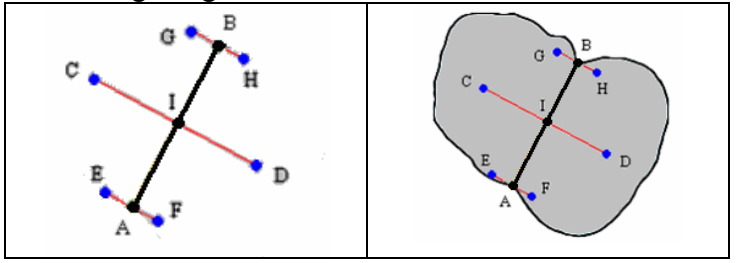

Figure 4. Overlapping template

The aggregating template (see. Figure 5) illustrates the fact that in aggregating areas, there is a narrow passage. The distances $\mathrm{CD}, \mathrm{EA}=\mathrm{AF}=\mathrm{GB}=\mathrm{BH}$ are also evaluated by observing a high number of nuclei. The angles are measured in function of tangent vectors left and right of concavity points $\mathrm{A}$ and $\mathrm{B}$.

As the template could be too rigid, some flexibility is introduced with the following rule: "Only one point among the points C, D, E, F, G, H could be outside the connected component".

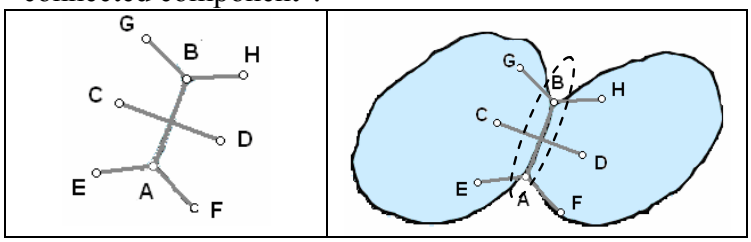

Figure 5. Aggregating template

If a potential segment of separation $[\mathrm{AB}]$ fits the overlapping template, the aggregating template, which is more restrictive, is then tested.

If the aggregating template is fitted, the segment $[\mathrm{AB}]$ is validated as a line of separation between nuclei in contact.
On the contrary, it could be validated as a segment of separation between overlapping nuclei. But, in some cases of very big and lobulated nucleus, a potential segment of separation might fit the overlapping template, though there is no overlapping area (see Figure 6b).

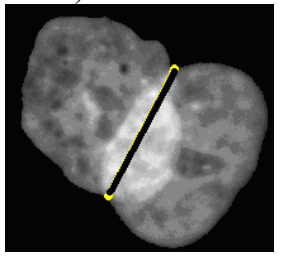

a

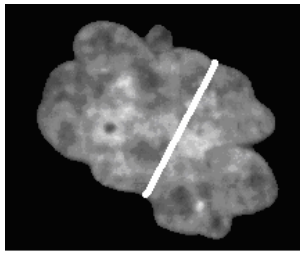

b
Figure 6. Potential segment separation fit overlapping template in case of (a)overlapping nuclei (b)an abnormal nucleus

Therefore, in order to distinguish these two configurations, a study of the grey levels intensity along $[\mathrm{AB}]$ is performed. In case of overlapping nuclei, the grey level intensity is far higher in the area of overlapping than the mean intensity of the connected component. In case of a big lobulated nucleus, no significant variation of grey levels can be observed, then the potential segment of separation is rejected.

\subsection{Watershed transform}

If the connected component includes several nuclei, they have to be separated. The watershed transform is then used. The critical step of this transform is the setting of the markers which are the starting points of flooding.

If the nuclei have been identified at the previous step as aggregating nuclei, a marker is set in the middle of the regions located on both sides of the validated segment of separation. If the nuclei overlap, there will be one more marker in the overlapping area along the segment $[\mathrm{AB}]$ (see Figure 7). Then the gradient image is considered as a topographical surface, which is flooded from the markers.
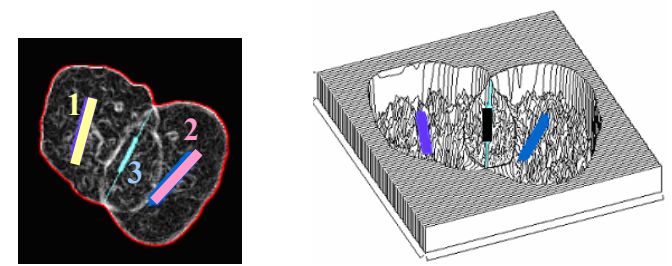

Figure 7. Initializing step of the watershed transform in case of overlapping nuclei

Lastly, a post-processing is applied to connect each region to the corresponding nucleus (see Figure 8): regions 1 and 3 form the first nucleus, and regions 2 and 3 the second one. 

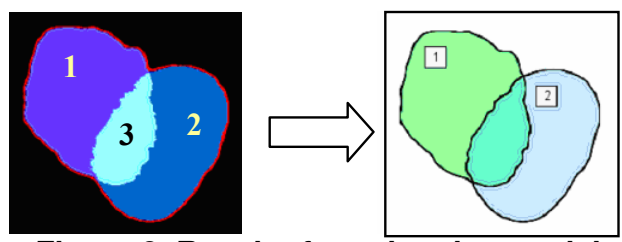

Figure 8. Result of overlapping nuclei segmentation

\section{Results}

This method has been tested and evaluated on images that contain 345 nuclei. About $76.23 \%$ of these nuclei are isolated, while $16.81 \%$ are overlapping and $6,96 \%$ aggregated.

Table 1: Rate of well-segmented nuclei in terms of number of nuclei

\begin{tabular}{|c|c|c|c|}
\hline $\begin{array}{c}\text { Isolated } \\
\text { Nuclei }\end{array}$ & $\begin{array}{c}\text { Aggregating } \\
\text { Nuclei }\end{array}$ & $\begin{array}{c}\text { Overlapping } \\
\text { Nuclei }\end{array}$ & Total \\
\hline 263 & 23 & 45 & 331 \\
\hline $100 \%$ & $95,83 \% \%$ & $77,59 \%$ & $95.94 \%$ \\
\hline
\end{tabular}

The results (see Table 1) show that $95.94 \%$ of nuclei are well segmented in terms of number of nuclei, according to biological experts. Furthermore, no minor deformation is missed. As these minor deformations play a fundamental role in drawing the right conclusions about shape normality, it is important not to miss them at the segmentation step.

In case of overlapping nuclei, most of the errors are due to the fact that only one point of high concavity is detected (see Figure 9), therefore no segment of separation can be extracted.

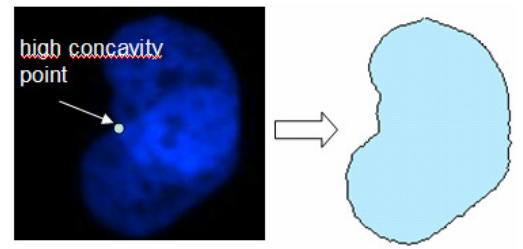

Figure 9. Example of overlapping nuclei where only 1 high concavity point is detected

\section{Conclusion}

In this paper we have presented a method, based on the watershed transform that can deal with aggregating/overlapping nuclei. The critical step of this transform is the initializing step. Prior information about nuclei is used to improve the selection of the best markers from which the flooding will start. The first results are quite encouraging but need to be validated on a larger set of data.

Future works will be devoted to the introduction of a formal learning step in order to obtain optimal aggregating/ overlapping templates based on biological knowledge extracted as rules or image samples. This would increase the fidelity in global applicability of the method.

\section{References}

[1] F. Cloppet, J.M. Oliva, G. Stamon, Angular Bisector Network, a Simplified Generalized Voronoï Diagram: Application to Processing Complex Intersections in Biomedical Images, IEEE Transactions on Pattern Analysis and Machine Intelligence, vol $22 \mathrm{n}^{\circ} 1$, janvier 2000, p120-128.

[2] M. Kass, A. Witkins, D. Terzopoulos, Snakes: Active Contours Models, International Journal of Computer Vision (1), pp. 321-331, 1988.

[3] J. Sethian, Curvature and the evolution of fronts, Communications in Mathematical Physics 101(4), pp. 487-499, 1985.

[4] L. Vincent, P. Soille, Watershed in digital spaces, an efficient algorithm based on immersion simulation, IEEE Transactions on Pattern Analysis Machine Intelligence 13(6), pp. 583-598, 1991.

[5] C. Zimmer and J.C. Olivo-Marin, Coupled Parametric Active Contours, IEEE Transactions on Pattern Analysis Machine Intelligence 27(11), pp. 1838-1842, 2005.

[6] B.Zhang, C. Zimmer and J.C. Olivo-Marin, Tracking fluorescent cells with coupled geometric active contours, IEEE International Symposium on Biomedical Imaging vol.1 pp. 476-479, 2004.

[7] L.A. Vese, T. Chan, A Multiphase level Set Framework for Image Segmentation Using the Mumford and Shah Model, International Journal of Computer Vision 50(3), pp. 271-293, 2002.

[8] J. AF Costa, ND Mascarenhas, and ML De Andrade Netto, Cell nuclei segmentation in noisy images using morphological watersheds, Proc. SPIE vol. 3164, pp. 314-324, 1997.

[9] Xiaowei Chen; Xiaobo Zhou; Wong, S.T.C., Automated segmentation, classification, and tracking of cancer cell nuclei in time-lapse microscopy, IEEE Transactions on Biomedical Engineering, Volume 53, Issue 4, April 2006 Page(s): 762 - 766

[10] V.Grau, A. Mewes, M. Alcañiz, R. Kkinis, S. Warfield, Improved watershed transform for medical image segmentation using prior information, IEEE Transactions on Medical Imaging 23(4), pp. 447-458, 2004.

\section{Acknowledgements}

The authors would like to thank N. Levy, P. Cau, C. Navarro and S. Perreira (both at Inserm U910 "Génétique Médicale et Développement", Faculté de Médecine de la Timone, 13385 Marseille Cedex 05) for given images, many useful suggestions and inspiring conversations and for their expertise. 